\title{
State Anger and the Risk of Injury: A Case-Control and Case-Crossover Study
}

\author{
Daniel C. Vinson, MD, MSPH \\ Vineesha Arelli, BS \\ 'Department of Family and Community \\ Medicine, University of Missouri-Columbia, \\ Columbia, Mo \\ ${ }^{2}$ School of Medicine, University of Missouri- \\ Columbia, Columbia, Mo
}

Conflicts of interest: none reported

\section{CORRESPONDING AUTHOR}

Daniel C. Vinson, MD, MSPH

Department of Family and Community

Medicine

University of Missouri-Columbia

M231 Medical Sciences Building

Columbia, MO 65212

VinsonD@health.missouri.edu

\begin{abstract}
PURPOSE Previous studies have examined anger at a given moment (state anger) and proxies for injury, or anger as a trait and injuries per se. Findings have been inconsistent. We sought to define further the relationship between state anger and risk of injury.
\end{abstract}

METHODS We conducted a case-control and case-crossover study in all 3 emergency departments in 1 county in Missouri. Cases were patients seeking care for an acute injury. They were compared with 2 controls: the patient himself or herself 24 hours before, and an individual recruited by telephone from the community and matched for age-group, sex, and time. Self-reported anger was assessed with 3 Likert scale items. Anger just before the injury was compared in case-crossover analyses with the respondent's own level of anger 24 hours before, and in standard case-control analyses with community participants' level of anger at the same hour the same day of the week in a subsequent week.

RESULTS Of 2,517 patients, 2,446 provided data on anger just before the injury, and 2,117 reported data for 24 hours before injury. Of 1,856 community individuals, 1,533 provided complete data. Anger was prevalent. Of injured patients, $9 \%, 7 \%$, and $4 \%$ reported feeling "quite a bit" or "extremely" "irritable," "angry," and "hostile," respectively, just before injury. Odds ratios for risk of injury were notably higher for greater degrees of anger; for example, for "angry," they were 1.8 (95\% confidence interval, 1.1- 2.7) for "quite a bit" and 7.2 (3.9-13) for "extremely." Odds ratios in women were substantially lower than those in men. Anger was not associated with fall and traffic injuries, but anger was strongly associated with intentional injuries inflicted by another person in both men and women.

CONCLUSIONS High levels of self-reported state anger increase the risk of injury, especially among men, and specifically the risk of intentional injury in both sexes.

Ann Fam Med 2006;4:63-68. DOI: 10.1370/afm.390.

\section{INTRODUCTION}

A nger is a common, complex emotion that may be associated with health and illness ${ }^{1-4}$ and with injury risk. It can be considered as state anger, an episode of anger occurring at a specified time, and as trait anger, an aspect of personality. ${ }^{3,4}$ Findings in previous research into the relationship between state or trait anger and injury have been inconsistent.

Anger may increase the risk of injury. A cohort study of 100 drivers followed up for 2 weeks found associations between state anger and "near accidents ${ }^{15}$ but did not address injury as such. Two other cohort studies found associations with trait anger, one with motor vehicle crashes ${ }^{6}$ and another with football injuries. ${ }^{7}$ A 6 -year Finnish cohort study found baseline trait hostility associated with subsequent all-cause injury (odds ratio $[\mathrm{OR}]=1.5)$ among men, but not among women. ${ }^{8}$

On the other hand, anger may not be associated with injury. Casecontrol studies of injured rugby players ${ }^{9}$ and people with hand injuries ${ }^{10}$ 
found no association between trait anger and injury. In a survey of US drivers, acting on anger by engaging in threatening driving behaviors was associated with the person's overall crash experience, but the relationship between state anger and a specific crash event was not examined. ${ }^{11}$

The studies we found examined either trait anger and injury, or state anger and a proxy for injury. None examined state anger and the risk of an injury occurring during that episode of anger. We therefore undertook a case-control and case-crossover study to define better the relationship between state anger and injury.

\section{METHODS \\ Study Participants}

Cases were patients seeking care at any of the 3 emergency departments in Boone County, Mo, within 48 hours of an injury between February 1998 and March 2000; hereafter, they are referred to as injured patients. Structured interviews were conducted by trained research staff in the emergency departments during systematically selected times (covered shifts) and with more severely injured patients in the hospital generally within 2 days of injury. We included injuries that had an identified time of occurrence and a mechanism listed in the Supplementary Classification of External Causes of Injury and Poisoning (E code) in the International Classification of Diseases, 9th edition.

These injured patients were matched with 2 comparison groups. First, each injured patient's self-reported level of anger just before the injury was compared with the same person's anger at the same time the previous day, using a case-crossover design, ${ }^{12}$ which has been used to study alcohol and the risk of injury ${ }^{13}$ and anger and the risk of a myocardial infarction, ${ }^{14,15}$ but has not been used to study anger and injury.

Second, controls were recruited from the community by random-digit dialing and matched to injured patients from covered shifts by age-group, sex, and rural vs urban residence, hereafter, they are referred to as community participants. At the time of the interview, each community participant was further matched to a specific injured patient's injury event by day of week, and the interview then focused on emotions and other variables at the hour of injury. Interviews were conducted from October 1999 through September 2000, and were generally matched within 2 months of the injured patient's injury date 1 or 2 years later. Analyses compared the injured patient's level of anger just before the injury with the community participant's level of anger at the matched time. The response rate among community members was $47 \%$. Details of subject recruitment and enrollment are given in a previous report. ${ }^{13}$

\section{Data Collection}

Emotions were measured by self-report using 21 single words or short phrases. We chose 18 items from the Positive and Negative Affect Schedule (PANAS) ${ }^{16,17}$ and added 3 others. The 21 items assessed positive emotions (eg, "daring," "alert," "excited") and negative emotions (eg, "sad," "sleepy," "scared"). The items relating to externally directed anger were all from the original PANAS_-irritable," "angry," and "hostile." Answer options used the PANAS's original 5-point scale, from "not at all" to "extremely." In selecting items, our goal was not to measure positive and negative affect as global constructs, but rather to measure different emotional states separately. Traits were not assessed, and questions addressed only the moments specified.

Interviews began with a review of the injury event for injured patients or, for community participants, activity at the matched day and hour. For both, we asked where they were, what they were doing, and whom they were with. Injured patients were asked these questions about location and activity a second time, focusing on the time 24 hours before the injury. Participants then responded to each of the 21 affect items based on their emotional state at the time in question. Injured patients went through the 21 items twice, answering how they felt just before injury and 24 hours before. Community participants went through the list only once, focusing on the matched time.

All participants reported their alcohol consumption in the 6 hours before the injury in number of drinks. One drink was defined as 12 ounces of beer, 5 ounces of wine, or 1.5 ounces of liquor. Injured patients' attributions of responsibility for their injury were assessed with 2 items scored on ordinal 5-point scales: "How much do you feel you were responsible for your injury?" and "How much do you feel someone else was responsible?"

\section{Statistical Analyses}

The unit of analysis was an injury event. Odds ratios and $95 \%$ confidence intervals were computed using conditional logistic regression, ${ }^{18}$ matching on individual in case-crossover analyses and on age-sex-time strata in case-control analyses. All measures of anger were skewed, $63 \%$ of injured patients responded "not at all" to all 3 items; therefore, to determine odds ratios associated with different levels of anger, we used the Stata interaction expansion ( $x i$ ) command, which assumes the variables are categorical, to create series of dummy variables in the conditional logistic regression analyses. For analyses of smaller subgroups, we dichotomized the anger variables into any self-reported anger vs none. 


\section{RESULTS} Participants

Table 1 shows demographic information about the participants. Of the 2,517 injured patients, 644 (26\%) were injured in a fall, $601(24 \%)$ were in a traffic or motor vehicle incident, $290(12 \%)$ were cut with a sharp instrument, 277 (11\%) were injured during sports, 103 (4\%) had an intentional injury inflicted by another person, and 19 (1\%) had a self-inflicted injury. The rest were injured by a variety of other mechanisms. The median time from injury to interview was 3.5 hours, with an interquartile range of 1.6 to 17.9 hours.

Of the 2,517 enrolled injured patients, 2,446 (97\%) provided data on all anger variables for the time just before injury, and 2,117 $(84 \%)$ provided data for the time 24 hours before injury; 332 (13\%) were asleep then. Of 1,856 community participants, between 7 and 10 persons were unable to answer a given affect item, and $306(17 \%)$ were asleep at the matched time ${ }_{i}$ in all, 1,533 provided complete data.

\section{Anger Variables}

Descriptive data on the affect variables for injured patients just before injury are given in Table 2. At this reference time, $33.0 \%$ of community participants reported some degree of irritability, $11.8 \%$ reported feeling angry, and $8.0 \%$ reported feeling hostile.

Among injured patients, all 3 anger variables were significantly $(P<.006)$ less commonly endorsed among those with traffic or fall injuries. For example, 13\% of those with traffic injuries reported some degree of "angry," compared with $20 \%$ of those with nontraffic injuries. Among community participants, at the matched time, $17 \%$ reported feeling at least moderately "irritable," $5 \%$ at least that degree of "angry," and $4 \%$ at least that degree of "hostile."

Of the 2,517 injured patients, 332 were asleep 24 hours before their injury, contributed no data for the comparison (control) time, and were omitted from case-crossover analyses. They were more likely to report anger at the time of injury than those who were awake 24 hours before injury, but the differences were small. In only one such comparison, "angry," was the difference statistically significant: $22 \%$ of those who were asleep 24 hours before their injury reported some degree of "angry," compared with $18 \%$ of those who were awake 24 hours before injury $(P=.04)$. The other absolute differences were $3 \%$ or $4 \%$, with $P$ values of .2 or greater.

\section{Anger and Risk of Injury}

In case-crossover analyses, higher levels of all anger variables were significantly associated with increased injury risk among men and women combined (Table 3). For men, the odds ratios were significant for "irritable" at levels of "quite a bit" or "extremely," for "angry" at "moderately" and above, and for all levels of "hostile." The odds ratio in men for the answer "extremely" for "irritable" was 4.8 (95\% CI, 2.6-8.8); for "angry," 7.3 (3.4-16); and for "hostile," 12 (3.4-40). Associations were smaller for women, among whom the 3 anger variables were all significantly associated with injury only at the level of "extremely," with odds ratios of 3.5 (95\% CI, 1.6-7.8) for "irritable," 7.4 (2.6-21) for "angry," and 4.3 (1.2-16) for "hostile." Subgroup analyses for African Americans $(n=232)$ and for whites $(n=2,138)$ showed no significant differences. 


\begin{tabular}{|c|c|c|c|c|}
\hline Emotion & $\begin{array}{c}\text { A Little } \\
\text { OR }(95 \% \mathrm{Cl})\end{array}$ & $\begin{array}{l}\text { Moderately } \\
\text { OR (95\% CI) }\end{array}$ & $\begin{array}{l}\text { Quite a Bit } \\
\text { OR }(95 \% \mathrm{Cl})\end{array}$ & $\begin{array}{c}\text { Extremely } \\
\text { OR }(95 \% \mathrm{Cl})\end{array}$ \\
\hline \multicolumn{5}{|c|}{$\begin{array}{l}\text { Case-crossover } \\
\text { analyses }\end{array}$} \\
\hline Irritable & $1.1(0.9-1.4)$ & $1.0(0.7-1.3)$ & $1.7(1.2-2.3)$ & $4.2(2.6-6.9)$ \\
\hline Angry & $1.1(0.8-1.4)$ & $1.9(1.3-2.9)$ & $1.8(1.1-2.7)$ & $7.2(3.9-13)$ \\
\hline Hostile & $1.3(1.0-1.9)$ & $2.2(1.3-3.5)$ & $2.7(1.5-4.7)$ & $7.9(3.3-19)$ \\
\hline \multicolumn{5}{|c|}{$\begin{array}{l}\text { Case-control } \\
\text { analyses }\end{array}$} \\
\hline Irritable & $0.8(0.6-1.0)$ & $0.7(0.5-0.9)$ & $1.1(0.8-1.6)$ & $1.9(1.2-3.1)$ \\
\hline Angry & $1.1(0.8-1.5)$ & $1.6(1.0-2.4)$ & $2.1(1.2-3.7)$ & $4.6(2.6-8.2)$ \\
\hline Hostile & $1.4(1.0-2.0)$ & $1.0(0.7-1.7)$ & $2.3(1.2-4.6)$ & $6.1(2.4-16)$ \\
\hline
\end{tabular}

another person (as reported by the injured patient) were high and generally statistically significant. Dichotomizing each anger variable into any level of the particular emotion vs none, the odds ratios for men were 5.7 (95\% $\mathrm{CI}$, 1.7-19) for "irritable," 7 (2.1-23) for "angry," and 20 (2.7-150) for "hostile." For women, they were 11 (1.4-85) for "irritable," 14 (1.8106) for "angry," and 11 (1.4-85) for "hostile."

\section{Responsibility for Injury}

Injured patients who were angered by the injury may have projected

In case-control analyses, results were similar (Table 3). Associations were again stronger for men, among whom the odds ratios for the answer "extremely" were 3.5 (95\% CI, 1.7-7.5) for "irritable," 8.2 (3.2-21) for "angry," and 6.2 (1.8-21) for "hostile". Among women, only the odds ratios for "angry" and "hostile" were significant, and then only at the highest level ("extremely"): 2.8 (1.3-5.9) for "angry" and 6.0 (1.3-27) for "hostile." More injured patients $(10.4 \%)$ than community participants $(6.7 \%)$ were African American, but controlling for race in case-control analyses did not significantly affect the associations between anger and injury risk.

\section{Mechanism of Injury}

In subgroup analyses by mechanism of injury, case-crossover analyses of traffic injuries and falls showed trends toward greater risk at greater levels of anger, but odds ratios were lower than in analyses including all injured patients and generally not significant. For example, at the level of "extremely," "angry" had an odds ratio of 4.2 (95\% CI, 0.9-20) for traffic injuries, and an odds ratio of $2.9(0.7-12)$ for fall injuries. Case-control analyses showed odds ratios significantly lower than 1 for traffic injuries for most levels of "irritable" and the lowest level of "angry," and no odds ratio for traffic injuries was significantly greater than 1 . No case-control odds ratios for fall injuries was significant. In case-control analyses for angry, the odds ratio for the answer "extremely" was 4.6 (95\% CI, 0.8-28) for traffic injuries and 2.8 (0.7-11) for fall injuries. The case-crossover and case-control findings regarding traffic injuries changed little when the analyses were limited to those injured patients who were driving the vehicle and whose vehicle was moving at the time of the injury, or to those who reported feeling responsible for their own injury.

In both case-crossover and case-control analyses, the odds ratios for injuries intentionally inflicted by that postevent anger back to preinjury time, distorting their perception of their pre-event anger. One factor that may have provoked such postinjury anger could have been the perception that someone else was responsible for the injury. We therefore did subgroup analyses, including among the injured patients only those who reported that no one else was responsible at all for their injury $(n=1,517)$. In case-crossover analyses, the associations were significant only for "extremely" "irritable" (odds ratio $=3.8,95 \% \mathrm{CI}, 1.9-7.4)$ and "angry" (4.9, 2.2-11); other odds ratios were between 0.8 and 1.6 and not significant. In case-control analyses, only the odds ratio for "angry" and the answer "extremely" was significantly greater than $1(2.9,1.5-5.6)$.

\section{Drinking and Injury}

Drinking during the previous 6 hours was strongly associated with injury risk. ${ }^{13}$ Among injured patients, the number of drinks in the 6 hours before the injury and the anger variables were correlated, with Spearman coefficients of 0.08 (for "irritable") to 0.19 (for "hostile") $(P$ values all <.001). Entering the number of drinks and each anger variable separately into case-crossover models, the odds ratios changed little. Education, marital status, employment status, and insurance status likewise had little effect on the findings in case-control analyses, and of course were matched in case-crossover comparisons.

\section{DISCUSSION}

In this study, emotions reflecting externally directed anger were common. The prevalence of anger among injured patients was as folows: $31.7 \%$ reported some degree of "irritable" just before the injury, 18.1\% reported feeling "angry," and 13.2\% reported feeling "hostile". More surprising was the prevalence of anger among the community participants, for whom the refer- 
ence time was essentially random: 33.0\% reported some degree of "irritable," $11.8 \%$ reported feeling "angry," and $8.0 \%$ reported feeling "hostile." Although much of that self-reported anger was mild, $5 \%$ of community participants reported feeling at least moderately "angry" and $4 \%$ reported feeling at least moderately "hostile."

Anger was associated with injury in this study primarily at higher levels of anger. In case-crossover and case-control analyses, respectively, odds ratios for the answer "extremely" were 4.2 and 1.9 for "irritable," 7.2 and 4.6 for "angry," and 7.9 and 6.1 for "hostile." The associations were stronger in men than in women.

The association between anger and injury varied substantially by injury mechanism. Traffic and fall injuries were generally not associated with anger in either case-control or case-crossover analyses. This finding is consistent with a Finnish cohort study that found no association between (trait) anger and traffic-related injuries. ${ }^{8}$ Anger while driving may not be as common as lay press reports might suggest; it was significantly less common among patients with traffic injuries in our study. Acting on that anger in ways likely to cause injury (eg, deliberately running into another vehicle) may also be uncommon. ${ }^{11}$ Even among those injured patients who acknowledged some responsibility for their own injury, the odds ratios were still close to 1 and not significant.

In contrast, injuries intentionally inflicted by another person were strongly associated with anger in both case-crossover and case-control analyses. We do not have data on the injured person's assessment of the assailant's affect just before the injury or self-assessment by the assailant; in addition, our findings do not clarify the pathways by which one's anger might increase one's risk of being injured. The findings do, however, indicate an association that may guide development of injury prevention strategies.

Self-reports of emotional states just before injury may have been biased, especially by anger aroused by a belief that someone else was responsible for causing the injury. When we included in the analyses only injured patients who reported no such attribution, the anger-injury association was substantially less. This finding could mean that the self-reports on which our analyses relied are unreliable, but 2 arguments can be made against that conclusion. First, those who ascribe responsibility for their injury to someone else might be expected to underreport rather than overreport their preinjury anger, to further deflect blame to the other person. Second, an external attributional style (a tendency to see others as responsible for one's problems) is associated with anger. ${ }^{19,20}$ We would therefore expect the bias to be in the opposite direction: injured patients with an external attributional style may have been more likely both to actually be angry before their injury and to ascribe responsibility for their injury to someone else, whether correctly or not. If this reasoning is correct, omitting from the analyses patients who blamed someone else would exclude persons who actually had preinjury anger, weakening the association between anger and injury.

Recalling emotional states 24 hours before injury (for injured patients) and at a random time the day before (for community participants) may have been more difficult than recall (by injured patients) of emotions right before injury. Anticipating this problem, we tried to facilitate accurate recall by asking participants to recall where they were, what they were doing, and whom they were with. In addition, more salient emotions such as anger may have been recalled more easily.

Anger is a complex emotion. In assessing it, we used only 3 items without definition or clarification, each self-reported on a 5 -point Likert scale. Measurement error and misclassification bias may have occurred; however, we used 3 of the 6 original 60 PANAS items related to anger (the ones we omitted were "scornful," "disgusted," and "loathing"), ${ }^{17}$ and we used its original answer options. We furthermore believe it is reasonable to assume that injured patients defined terms such as "angry" the same way for both case time and control time, minimizing misclassification bias at least in case-crossover analyses. Finally, our reliance on simple assessment of anger is not unprecedented. The case-crossover studies of anger and myocardial infarction both used a single item with a 7 -point Likert scale answer to assess state anger. ${ }^{14,15}$

In case-crossover analyses, data were missing for $13 \%$ of the injured patients because they were asleep at the matched time the day before, and the effects of these missing data on the findings are uncertain. Among those without data for the day before, however, self-reported anger just before the injury was slightly more common than among those who were awake 24 hours before injury; therefore, the case-crossover odds ratios reported here may be underestimated.

Because each injured person was compared with himself or herself the day before, all stable personal characteristics were perfectly matched in case-crossover analyses. These characteristics include trait anger and other personality constructs, to the extent they are invariant from one day to the next. Given the low response rate among the community and the possibility of residual unmeasured confounding in the case-control analyses, the case-crossover analyses may provide more reliable estimates of the anger-injury association.

For some mechanisms of injury, especially intentional injury, anger is significantly associated with injury risk. The association is stronger among men than among women. Because of the sources of potential bias outlined 
above, these findings should be considered tentative. Studying anger at a given moment and the risk of an injury occurring at that moment is inherently challenging methodologically, however; measuring anger prospectively and assessing its relationships with actual injury events is not feasible. These findings, though tentative, provide provocative insights into the possible complex associations between state anger and injury risk.

To read or post commentaries in response to this article, see it online at http://www.annfammed.org/cgi/content/full/4/1/63.

Key words: Anger; wounds and injuries; violence; behavior; psychosocial factors

Submitted January 22, 2005; submitted, revised, June 27, 2005; accepted July 5, 2005.

A version of this report was presented at the Society of Teachers of Family Medicine Annual Spring Conference, May 12, 2004, Toronto, Canada.

Funding support: The study was funded by a grant from the National Institute on Alcohol Abuse and Alcoholism (R01 AA11078). Additional support was provided by the Department of Family and Community Medicine at the University of Missouri-Columbia through the Opal Lewis Fund for alcohol research and the Center for Family Medicine Science, which was supported by a grant from the American Academy of Family Physicians.

Acknowledgments: Malcolm Maclure, ScD, School of Health Information Science, University of Victoria, Victoria, British Columbia, and Gordon S. Smith, MB, ChB, MPH, then at the Center for Injury Research and Policy, School of Hygiene and Public Health, the Johns Hopkins University, Baltimore, Md, helped with design and interpretation of the larger study that provided the data examined here. Nancy Mabe, MD, helped with design of the preliminary study on which the current work was built. Interviews of injured patients were conducted by Carol Reidinger; Carey Smith; Ciprian Crismaru, MD; Amelia Devera-Sales, MD; Indira Gujral; Kari Gilmore; and Lindsay Wiles, Aneesh Tosh, Stephen Griffith, Darin Lee, Greg Morlin, and Rebecca Shumate, who were medical students at the time. Data management was done by Sandy Taylor; Darla Horman; Robin Kruse, PhD; and Carol Reidinger. Telephone interviews of community participants were conducted by Research Triangle Institute, Inc, Research Triangle Park, NC. We are very grateful to our colleagues in the emergency departments who helped make this study possible.

\section{References}

1. Vahtera J, Kivimaki M, Koskenvuo M, Pentti J. Hostility and registered sickness absences: a prospective study of municipal employees. Psychol Med. 1997;27:693-701.

2. Ranchor AV, Sanderman R, Bouma J, Buunk BP, van den Heuvel WJ. An exploration of the relation between hostility and disease. J Behav Med. 1997;20:223-240.
3. Spielberger CD, Sydeman SJ, Owen AE, Marsh BJ. Measuring anxiety and anger with the State-Trait Anxiety Inventory (STAI) and the StateTrait Anger Expression Inventory (STAXI). In: Maruish ME, ed. The Use of Psychological Testing for Treatment Planning and Outcomes Assessment. 2nd ed. Mahwah, NJ: Lawrence Erlbaum Associates; 1999:9931021.

4. Mayne TJ, Ambrose TK. Research review on anger in psychotherapy. J Clin Psychol. 1999;55:353-363.

5. Underwood G, Chapman P, Wright S, Crundall D. Anger while driving. Transportation Res F: Traffic Psychol Behav. 1999;2F:55-68.

6. Norris FH, Matthews BA, Riad JK. Characterological, situational, and behavioral risk factors for motor vehicle accidents: a prospective examination. Accid Anal Prev. 2000;32:505-515.

7. Thompson NJ, Morris RD. Predicting injury risk in adolescent football players: the importance of psychological variables. J Pediatr Psychol. 1994;19:415-429.

8. Romanov K, Hatakka M, Keskinen E, et al. Self-reported hostility and suicidal acts, accidents, and accidental deaths: a prospective study of 21,443 adults aged 25 to 59. Psychosom Med. 1994;56:328-336.

9. Garraway WM, Lee AJ, Macleod DA, et al. Factors influencing tackle injuries in rugby union football. Br J Sports Med. 1999;33:37-41.

10. Chau N, d'Houtaud A, Gruber M, et al. Personality self-representations of patients with hand injury, and its relationship with work injury. Eur J Epidemiol. 1995;11:373-382.

11. Wells-Parker E, Ceminsky J, Hallberg V, et al. An exploratory study of the relationship between road rage and crash experience in a representative sample of US drivers. Accid Anal Prev. 2002;34:271-278.

12. Maclure M, Mittleman MA. Should we use a case-crossover design? Annu Rev Public Health. 2000;21:193-221.

13. Vinson DC, Maclure M, Reidinger C, Smith GS. A population-based case-crossover and case-control study of alcohol and the risk of injury. J Stud Alcohol. 2003;64:358-366.

14. Mittleman MA, Maclure M, Sherwood JB, et al. Triggering of acute myocardial infarction onset by episodes of anger. Determinants of Myocardial Infarction Onset Study Investigators. Circulation. 1995;92:1720-1725.

15. Moller J, Hallqvist J, Diderichsen $F$, et al. Do episodes of anger trigger myocardial infarction? A case-crossover analysis in the Stockholm Heart Epidemiology Program (SHEEP). Psychosom Med. 1999;61:842-849.

16. Watson D, Clark LA, Tellegen A. Development and validation of brief measures of positive and negative affect: the PANAS scales. J Pers Soc Psychol. 1988;54:1063-1070.

17. Watson D, Clark LA. The PANAS-X: Manual for the Positive and Negative Affect Schedule-Expanded Form. Ames, lowa: University of lowa; 1994

18. Stata statistical software: Release 8.1. College Station, Tex: Stata Corporation; 2003.

19. Carmony TM, DiGiuseppe R. Cognitive induction of anger and depression: the role of power, attribution, and gender. J Rational-Emotive Cogn Behav Ther. 2003;21:105-118.

20. Neumann R. The causal influences of attributions on emotions: a procedural priming approach. Psychol Sci. 2000;11:179-182. 